\title{
Spiroergometrie bei Patienten mit chronisch obstruktiver Lungenerkrankung - multizentrischer Vergleich von zwei Belastungsprotokollen
}

\author{
Cardiopulmonary Exercise Testing (CPET) in Severe COPD - A Multicentre \\ Comparison of Two Test Protocols
}

Autoren

Institute
R. Ewert ${ }^{1}$, S. Gläser ${ }^{1}$, J. Winkler² ${ }^{2}$, H. Schrader ${ }^{3}$, B. G.Trümper ${ }^{4}$, P.-U. Haase ${ }^{5}$, A. Obst ${ }^{1}$, G. Hoheisel ${ }^{6}$

Die Institutsangaben sind am Ende des Beitrags gelistet. eingereicht 17.2 .2012 akzeptiert nach Revision 25. 3. 2012

\section{Bibliografie}

Dol http://dx.doi.org/

10.1055/s-0032-1309732

Online-Publikation: 23.5.2012

Pneumologie 2012; 66: 402-407

(c) Georg Thieme Verlag KG

Stuttgart · New York

ISSN 0934-8387

Korrespondenzadresse Prof. Dr. med. Ralf Ewert Ernst-Moritz-Arndt-Universität Greifswald, Universitätsmedizin Klinik für Innere Medizin B Friedrich-Loeffler-Str. 23a 17489 Greifswald ewert@uni-greifswald.de

\section{Zusammenfassung \\ $\nabla$}

Die Spiroergometrie erlaubt bei COPD-Patienten eine valide Beurteilung der kardiopulmonalen Belastbarkeit und die Beschreibung vorliegender Limitierungen. Während die Messungen von Ventilation und Gasaustausch weitgehend standardisiert sind, existiert keine allgemeine Empfehlung bezüglich des zu wählenden Belastungsprotokolls. Wir haben daher multizentrisch zwei Belastungsprotokolle in pneumologischen Facharztpraxen verglichen. Es wurden 90 COPD-Patienten der GOLD-Stadien II-IV mittels symptomlimitierter Fahrradbelastung mit zwei unterschiedlichen Rampenprotokollen (10 Watt vs. 16 Watt/min) innerhalb von 7 Tagen untersucht. Die Ergebnisse zeigen, dass eine höhere Steigerungsrate mit einer kürzeren Belastungszeit und höheren Leistung assoziiert ist. Alle anderen spiroergometrischen Parameter der Ventilation und des Gasaustausches zeigen keine signifikanten Veränderungen beim Vergleich beider Protokolle. In pneumologischen Praxen können COPD-Patienten der GOLD-Stadien II-IV sicher und valide mittels Spiroergometrie untersucht werden. Die Anwendung eines Rampenprotokolls mit Steigerung von 16 Watt/Minute kann empfohlen werden, zumal hierfür aktuelle Normwerte vorliegen.

\section{Einleitung}

$\nabla$

Bei Patienten mit chronisch obstruktiver Lungenerkrankung (COPD) kann der Schweregrad der kardiopulmonalen Belastungseinschränkung sowie die vorrangige Limitierung (ventilatorisch, kardial, muskulär) mittels der Spiroergometrie (cardiopulmonary exercise testing, CPET) gut bestimmt werden [1,2]. Die Ergebnisse der CPET sind bei Patienten mit COPD geeignet, die Wirksamkeit von therapeutischen Interventionen darzustellen [3-5] und Voraussagen zur Prognose [6

\section{Abstract \\ $\nabla$}

Cardiopulmonary exercise testing (CPET) allows a valid evaluation of cardiopulmonary function capability and the recognition of existing limitations in COPD patients. Whereas the measurement of the different parameters of CPET and the evaluation of the results are standardised, this does not apply to the protocols chosen. The aim of this study was to evaluate whether the results of two different exercise protocols were comparable in an outpatient multicentre setting. Ninety COPD patients stages II-IV according to the Gold classification were examined by means of symptom-limited exercise testing on a bicycle with two different ramp protocols (10 Watts vs. 16 Watts/min) within seven days. The results show that a higher acceleration rate of the load was associated with shorter exercise duration and higher achieved exercise capacity. Gas exchange and ventilatory parameters did not show significant differences on comparing both protocols. In pulmonary practices COPD patients of the Gold stages II-IV can be examined safely and with validity by means of CPET. The application of a ramp protocol with a stepwise increase of 16 Watts/minute can be recommended, particularly as for this regimen standardised normal values are available.

-9] abzuleiten. Zunehmend findet die CPET auch Anwendung zur Begutachtung von pneumologischen Berufserkrankungen, u.a. für solche, die mit einer Atemwegsobstruktion einhergehen [10].

Während die Messung der kardialen, respiratorischen und metabolischen Parameter während der CPET gut standardisiert ist, trifft dies für die zur Verfügung stehenden Belastungsprotokolle nicht zu. Vor diesem Hintergrund sind bisherige Bemühungen zur Vergleichbarkeit verschiedener CPET-Protokolle bei COPD-Patienten zu verstehen 
[11-15]. Als ein Ergebnis dieser bisherigen Untersuchungen konnte gezeigt werden, dass bei der symptomlimitierten Ausbelastung die maximal erreichte Leistung wesentlich durch das gewählte Protokoll bestimmt wird. Dagegen zeigen die Daten für die Ventilation bzw. den Gasaustausch keine solche Abhängigkeit von der Art des gewählten Protokolls. Die bisher publizierten Studien zum Vergleich verschiedener CPET-Protokolle bei COPD-Patienten basieren jedoch a) nur auf kleinen Patientengruppen und b) sind monozentrisch in klinischen Einrichtungen durchgeführt worden.

Wir haben daher in einer multizentrischen, prospektiv randomisierten Studie (www.controlled-trials.com, ISRCTN22314061) mit COPD-Patienten in fünf deutschen pneumologischen Facharztpraxen untersucht, ob in Abhängigkeit des gewählten CPETProtokolls unterschiedliche Ergebnisse resultieren.

\section{Methoden}

Die prospektive Beobachtungsstudie wurde in fünf pneumologischen Fachpraxen unter Einbeziehung konsekutiver COPD-Patienten durchgeführt. Die Diagnose der COPD sowie die Schweregradeinteilung erfolgten nach den Kriterien der gültigen Leitlinie zur Diagnostik und Therapie der COPD [16]. Die Patienten waren zwischen 38-84 Jahre alt und standen unter einer stabilen medikamentösen Therapie seit mindestens vier Wochen. Kontraindikationen zur Teilnahme an der Studie waren: Fehlende schriftliche Einwilligung, signifikante linksventrikuläre Pumpfunktionsstörung ( $\mathrm{LVEF}<50 \%$ ), instabile koronare Herzerkrankung, Myokardinfarkt in den letzten 6 Monaten; klinisch relevante andere Lungenerkrankung (andere als COPD); relevante muskuloskeletale Störungen mit Einfluss auf die Belastbarkeit; Veränderungen der Dauermedikation sowie Exazerbation in den letzten 4 Wochen.

Das Studienprotokoll wurde durch die zuständige Ethikkommission positiv beurteilt, und jeder Patient gab seine schriftliche Einwilligung zur Durchführung der Untersuchung und wissenschaftlichen Auswertung der anonymisierten Daten.

In allen fünf Praxen wurde das Personal hinsichtlich der Durchführung der Untersuchungen eingewiesen und anhand von jeweils zwei COPD-Patienten (deren Daten nicht in die Auswertung einbezogen wurden) eine Qualitätssicherung vorgenommen. Die Auswertung der erhobenen CPET-Daten erfolgte doppelt durch unabhängige Befunder.

\section{Spirometrie und Bodyplethysmografie}

Bei allen Patienten wurde am Tag der CPET jeweils eine Spirometrie (Pneumotachografie) und Bodyplethysmografie [VIASYS Healthcare, Jaeger, Höchberg, Deutschland] entsprechend der gültigen Empfehlungen durchgeführt $[17,18]$, wobei die Untersuchung unter Beibehaltung der bisherigen Medikation erfolgte. Zur Bewertung wurden die etablierten alters- und geschlechtsspezifischen Normwerte verwendet [19].

\section{Spiroergometrie (CPET)}

Die symptomlimitierte kardiopulmonale Belastung wurde auf einem Fahradergometer (Ergometrics 900, Ergoline $\mathrm{GmbH}$, Deutschland) durchgeführt. Die methodischen Details der Messung sowie die Normwerte für ein Rampenprotokoll auf dem Fahrrad wurden kürzlich mitgeteilt [20], daher hier in Kürze nur einige wesentliche Beschreibungen. Die Sauerstoffaufnahme $\left(\mathrm{VO}_{2}\right)$, Kohlendioxidabgabe $\left(\mathrm{VCO}_{2}\right)$ und die Ventilation (VE) wur- den Atemzug für Atemzug gemessen (ZAN 600, NSpire Health $\mathrm{GmbH}$, Deutschland bzw. Oxycon Pro, Viasys HealthCare $\mathrm{GmbH}$, Deutschland). Die Spitzen-Sauerstoffaufnahme (peak $\mathrm{VO}_{2}$ ) wurde als höchster Wert in den letzten 10 Sekunden der Belastung ermittelt. Die perkutan gemessene Sauerstoffsättigung und das 12-Kanal-Elektrokardiogramm wurden kontinuierlich und der nichtinvasiv ermittelte Blutdruck in Ruhe, während jeder Stufe der Belastung und am Ende der Untersuchung gemessen. Die Blutgasanalyse erfolgte jeweils aus dem arterialisierten Kapillarblut des Ohrläppchens in Ruhe, an der anaeroben Schwelle und bei maximaler Belastung. Die maximale mögliche Ventilation (MVV) wurde wie folgt berechnet: $\mathrm{FEV}_{1}$-Wert (direkt vor der Untersuchung gemessen) $\times 41$ [21].

Die Untersuchung wurde jeweils mit einer Ruhephase von 3-5 Minuten, sitzend mit Maske auf dem Fahrrad, zur Messung der Ausgangswerte begonnen. Dann erfolgte eine kurze Phase (1 - 3 Minuten) des Tretens ohne Last („unloaded pedaling“), und nachfolgend wurde die Belastung mit einer Steigerung von jeweils 16 Watt pro Minute begonnen (Jones-Protokoll) [22]. Eine zweite Untersuchung wurde innerhalb einer Woche nach der ersten Untersuchung in vergleichbarer Weise durchgeführt, wobei die Steigerung mit jeweils 10 Watt pro Minute erfolgte (Protokoll 2). Zur Bewertung wurden die etablierten alters- und geschlechtsspezifischen Normwerte verwendet [23].

\section{Statistik}

Power-Analyse

Basis für die Power-Analyse ist der Vergleich von zwei unterschiedlichen Belastungsprotokollen im Sinne der Fragestellung. Zum Vergleich der Protokolle gingen wir von einer mit anderen Arbeiten vergleichbaren Variabilität von 6-10\% der zentralen Parameter (peak $\mathrm{VO}_{2}$, ventilatorische Parameter, Parameter der Atemeffizienz, Blutgase und Herzfrequenz) aus [24,25]. Die Anzahl der Patienten wurde so gewählt, dass Unterschiede mit einem zweiseitigen t-Test (Signifikanzniveau von 0,05) und mit einer Power von $>80 \%$ gewährleistet waren.

\section{Datenbearbeitung}

Sämtliche Daten wurden in Form einer Datenbank (Excel) erfasst. Kontinuierliche Daten wurden als Mittelwert und Standardabweichung angegeben. Mittelwert-Vergleiche erfolgten mittels Wilcoxon-Test. Der Vergleich innerhalb der Patientengruppe, d. h. zwischen den beiden Protokollen, erfolgte mit dem WilcoxonVorzeichen-Rang-Test. Zum Vergleich der beiden untersuchten Protokolle wurden zusätzlich für ausgewählte Parameter BlandAltman-Diagramme und Boxplots dargestellt. Ein p-Wert von $<0,05$ wurde als signifikant erachtet. Die statistischen Analysen wurden mit dem Programm SAS 9.1 (SAS Institute Inc., Cary, NC, USA) gerechnet und die Bland-Altman-Diagramme mithilfe des Programms MEDCal dargestellt.

\section{Ergebnisse}

\section{Patienten}

Die Daten zur Charakterisierung der Patienten (ein Patient wurde ausgeschlossen, da nur eine auswertbare CPET vorlag) sind in der - Tab. 1 enthalten. In den Praxen wurden zwischen 25 (Praxis 1) und 11 (Praxis 5) Patienten eingeschlossen. Hinsichtlich der Verteilung der GOLD-Schweregrade 3 bzw. 4 zeigten sich keine Unterschiede zwischen den Praxen $(p=0,87)$. Die Patienten waren im Mittel $65 \pm 9,6$ Jahre alt, und die COPD war im Mittel 
Tab.1 Demografische Daten der ausgewerteten COPD-Patienten ( $\mathrm{N}=90)$.

\begin{tabular}{|c|c|c|}
\hline Charakteristik & Prozent & $\begin{array}{l}\text { Mittelwert } \\
\text { ( } \pm \text { Standardabweichung) }\end{array}$ \\
\hline Anteil weiblicher Patienten (\%) & 16,7 & \\
\hline Alter (in Jahren) & & $65,6( \pm 9,6)$ \\
\hline COPD bekannt seit (in Jahren) & & $10,6( \pm 7,0)$ \\
\hline Exazerbationen (im letzten Jahr) & & $2,4( \pm 4,3)$ \\
\hline \multicolumn{3}{|l|}{ Raucher (\%) } \\
\hline Nie-Raucher & 6,7 & \\
\hline Raucher & 34,4 & \\
\hline Ex-Raucher & 58,9 & \\
\hline Ex-Raucher seit (in Jahren) & & $11,4( \pm 10,1)$ \\
\hline Packungsjahre/Pack-Years & & $36,8( \pm 19,2)$ \\
\hline Raucher & & $35,1( \pm 15,0)$ \\
\hline Ex-Raucher & & $37,9( \pm 21,3)$ \\
\hline \multicolumn{3}{|l|}{ GOLD-Stadien (\%) } \\
\hline 2 & 6,7 & \\
\hline 3 & 68,9 & \\
\hline 4 & 24,4 & \\
\hline
\end{tabular}

Angaben in Prozent bzw. als Mittelwert und Standardabweichung.

Tab. 2 Ausgewählte lungenfunktionelle Befunde.

\begin{tabular}{|llc|}
\hline Variable & N & Mittelwert $( \pm$ Standardabweichung) \\
\hline VC & 90 & $2,5( \pm 0,8)$ \\
\hline VC in Prozent & 90 & $63,1( \pm 13,9)$ \\
\hline FVC & 90 & $2,2( \pm 0,8)$ \\
\hline FVC in Prozent & 90 & $58,0( \pm 15,1)$ \\
\hline FEV $_{1}$ & 90 & $1,2( \pm 0,3)$ \\
\hline FEV $_{1}$ in Prozent & 90 & $39,6( \pm 8,3)$ \\
\hline Quotient $_{\text {FEV }} /$ VC & 90 & $48,3( \pm 9,6)$ \\
\hline Rtot & 89 & $0,6( \pm 0,2)$ \\
\hline Rtot in Prozent & 89 & $206,0( \pm 81,1)$ \\
\hline ITGV & 89 & $5,9( \pm 1,5)$ \\
\hline ITGV in Prozent & 89 & $171,1( \pm 41,1)$ \\
\hline
\end{tabular}

VC - Vitalkapazität; FVC - forcierte Vitalkapazität, FEV 1 - forciertes exspiratorisches Volumen der ersten Sekunde, Rtot - totale Resistance, ITGV - intrathorakales Gasvolumen.

seit $11 \pm$ 7,0 Jahren bekannt. Lediglich sechs Patienten hatten nie geraucht, und 53 (im Mittel seit 11 Jahren) hatten das Rauchen aufgegeben. Die inhalative medikamentöse Therapie wurde wie folgt durchgeführt (Doppelnennungen möglich): kurzwirksame inhalative Beta-Mimetika 96\% (86/90), langwirksame inhalative Beta-Mimetika 88\% (79/90), kurzwirksame inhalative Anticholinergika 58\% (52/89), langwirksame inhalative Anticholinergika 79\% (71/90) und inhalative Kortikosteroide 83\% (75/90) der Patienten. Zusätzlich erhielten 19 Patienten ein oral verfügbares Xanthin-Derivat, und lediglich bei zwei Patienten wurden zusätzlich orale Kortikoide ( $5 \mathrm{mg} / \mathrm{d}$ ) verabreicht.

\section{Lungenfunktionelle Befunde}

Eine Übersicht zu den lungenfunktionellen Befunden wird in der - Tab. 2 gegeben. Dabei wird deutlich, dass bei einem Patienten keine bodyplethysmografische Untersuchung vorlag.
Tab. 3 Vergleich ausgewählter CPET-Daten bei unterschiedlichen Protokollen.

\begin{tabular}{|c|c|c|c|}
\hline Variable & $\begin{array}{l}\text { Jones- } \\
\text { Protokoll }\end{array}$ & $\begin{array}{l}\text { 10-Watt- } \\
\text { Protokoll }\end{array}$ & P Wert \\
\hline Belastungsdauer (Minuten) & $4,5( \pm 1,6)$ & $5,5( \pm 2,0)$ & $<0,001$ \\
\hline $\mathrm{VO}_{2}$ an der AT (Liter/min) & $0,87( \pm 0,23)$ & $0,85( \pm 0,22)$ & 0,81 \\
\hline $\mathrm{VE} / \mathrm{VCO}_{2}$ an $\mathrm{AT}$ & $34,7( \pm 6,1)$ & $34,9( \pm 5,8)$ & 0,703 \\
\hline petCO ${ }_{2}$ an AT $(\mathrm{mmHg})$ & $35,6( \pm 6,7)$ & $35,7( \pm 6,4)$ & 0,928 \\
\hline petO $_{2}$ an AT $(\mathrm{mmHg})$ & $105,3( \pm 7,4)$ & $105,0( \pm 8,5)$ & 0,722 \\
\hline \multicolumn{4}{|l|}{ Maximale Belastung } \\
\hline Max. Leistung (Watt) & $78,2( \pm 23,6)$ & $67,1( \pm 19,5)$ & $<0,001$ \\
\hline Max. Ventilation (Liter) & $41,8( \pm 12,5)$ & $40,5( \pm 10,8)$ & 0,072 \\
\hline Max. VE/MVV & $0,9( \pm 0,2)$ & $0,9( \pm 0,3)$ & 0,274 \\
\hline Max. Vitalkapazität (Liter) & $1,4( \pm 0,4)$ & $1,4( \pm 0,4)$ & 0,760 \\
\hline Max. Atemfrequenz/min & $31,3( \pm 4,9)$ & $30,2( \pm 5,4)$ & 0,002 \\
\hline $\mathrm{VO}_{2}($ Liter/min $)$ & $1,1( \pm 0,3)$ & $1,1( \pm 0,3)$ & 0,913 \\
\hline $\mathrm{VO}_{2}$ (Prozent der Norm) & $59,7( \pm 14,3)$ & $60,1( \pm 15,5)$ & 0,917 \\
\hline $\mathrm{VO}_{2}(\mathrm{ml} / \mathrm{kg} / \mathrm{min})$ & $15,0( \pm 3,3)$ & $15,2( \pm 4,1)$ & 0,757 \\
\hline VE/VCO 2 slope & $31,8( \pm 5,4)$ & $31,6( \pm 5,1)$ & 0,423 \\
\hline Herzfrequenz $\left(\mathrm{min}^{-1}\right)$ & $120,2( \pm 17,5)$ & $121,2( \pm 16,3)$ & 0,843 \\
\hline Blutdruck (syst.) (mmHg) & $170,2( \pm 28,7)$ & $172,3( \pm 26,4)$ & 0,517 \\
\hline Blutdruck (diastol.) (mmHg) & $90,9( \pm 22,1)$ & $93,1( \pm 19,7)$ & 0,287 \\
\hline PH-Wert & $7,4( \pm 0,1)$ & $7,4( \pm 0,0)$ & 0,537 \\
\hline $\mathrm{PaCO}_{2}(\mathrm{mmHg})$ & $42,6( \pm 6,4)$ & $42,7( \pm 6,4)$ & 0,656 \\
\hline $\mathrm{PaO}_{2}(\mathrm{mmHg})$ & $67,5( \pm 12,5)$ & $68,9( \pm 11,7)$ & 0,013 \\
\hline $\mathrm{AaDO}_{2}(\mathrm{mmHg})$ & $36,0( \pm 10,8)$ & $33,1( \pm 10,1)$ & $<0,001$ \\
\hline PaetCO $_{2}(\mathrm{mmHg})$ & $8,1( \pm 4,8)$ & $8,2( \pm 4,4)$ & 0,636 \\
\hline
\end{tabular}

$\mathrm{VO}_{2}$ an der AT - Sauerstoffaufnahme an der anaeroben Schwelle; VE $/ \mathrm{VCO}_{2}$ - Atemäquivalent für $\mathrm{CO}_{2}$; petCO $\mathrm{CO}_{2}$ - endtidaler Partialdruck für $\mathrm{CO}_{2}$; petO $\mathrm{O}_{2}$ - endtidaler Partialdruck für $\mathrm{O}_{2} ; \mathrm{AaDO}_{2}$ - alveolo-arterielle Partialdruckdifferenz für Sauerstoff; PaetCO $\mathrm{C}_{2}$ - arteriell-alveoläre Kohlendioxiddifferenz; Angaben als Mittelwert ( \pm Standardabweichung).

\section{Werte der CPET}

\section{Auswertbarkeit}

Von den 91 Patienten waren bei 90 Patienten gepaarte Teste vorhanden, bei einem Patienten war eine Untersuchung aufgrund eines technischen Defekts nicht auswertbar. Bei Anwendung des Jones-Protokolls konnte bei 68 Patienten die anaerobe Schwelle sicher bestimmt werden. In 20/22 Fällen war der Abbruch der Untersuchung vor Erreichen der anaeroben Schwelle die Ursache. Bei Verwendung des Protokolls 2 konnte in 72 Fällen die anaerobe Schwelle bestimmt werden, wobei hier in 15/18 der Abbruch der Untersuchung vor Erreichen der anaeroben Schwelle erfolgte.

\section{Protokollvergleich}

Die Werte der CPET sind in Tab. 3 ausgewiesen. Die Patienten erreichten mit dem Jones-Protokoll eine signifikant höhere Leistung während einer signifikant kürzeren Belastungszeit (entspricht der Zeit ohne „unloaded pedaling“). In der $\bullet$ Abb. 1 wird die lineare Beziehung zwischen Belastungszeit und Leistung dargestellt, wobei die Steigung (slope) für das Jones-Protokoll im Vergleich zum Protokoll 2 größer ist.

Bei Betrachtung der maximal erreichten Sauerstoffaufnahme ( $\bullet$ Abb.2), der Sauerstoffaufnahme an der anaeroben Schwelle, der Atemeffizienz, dargestellt als $\mathrm{VE} / \mathrm{VCO}_{2}$ slope $(\bullet \mathrm{Abb} .3$ ), des Atemäquivalents für $\mathrm{CO}_{2}$ an der anaeroben Schwelle sowie weiterer Gasaustauschparameter zeigen sich im Vergleich der Protokolle keine signifikanten Unterschiede. Lediglich für die alveoloarterielle Partialdruckdifferenz für Sauerstoff $\left(\mathrm{AaDO}_{2}\right)$ zeigt sich ein geringer, jedoch signifikanter Unterschied. 


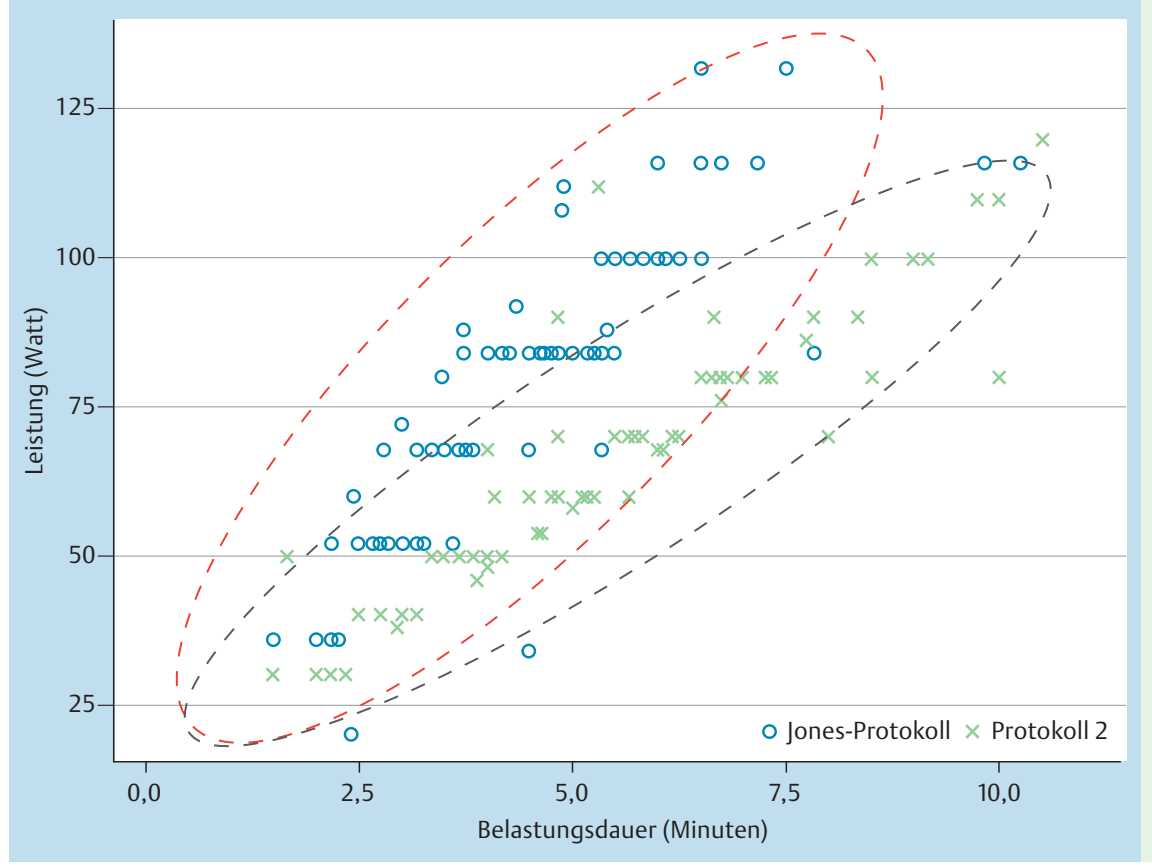

Abb.1 Darstellung der linearen Beziehung zwischen Belastungszeit und Leistung für die beiden gewählten Protokolle.

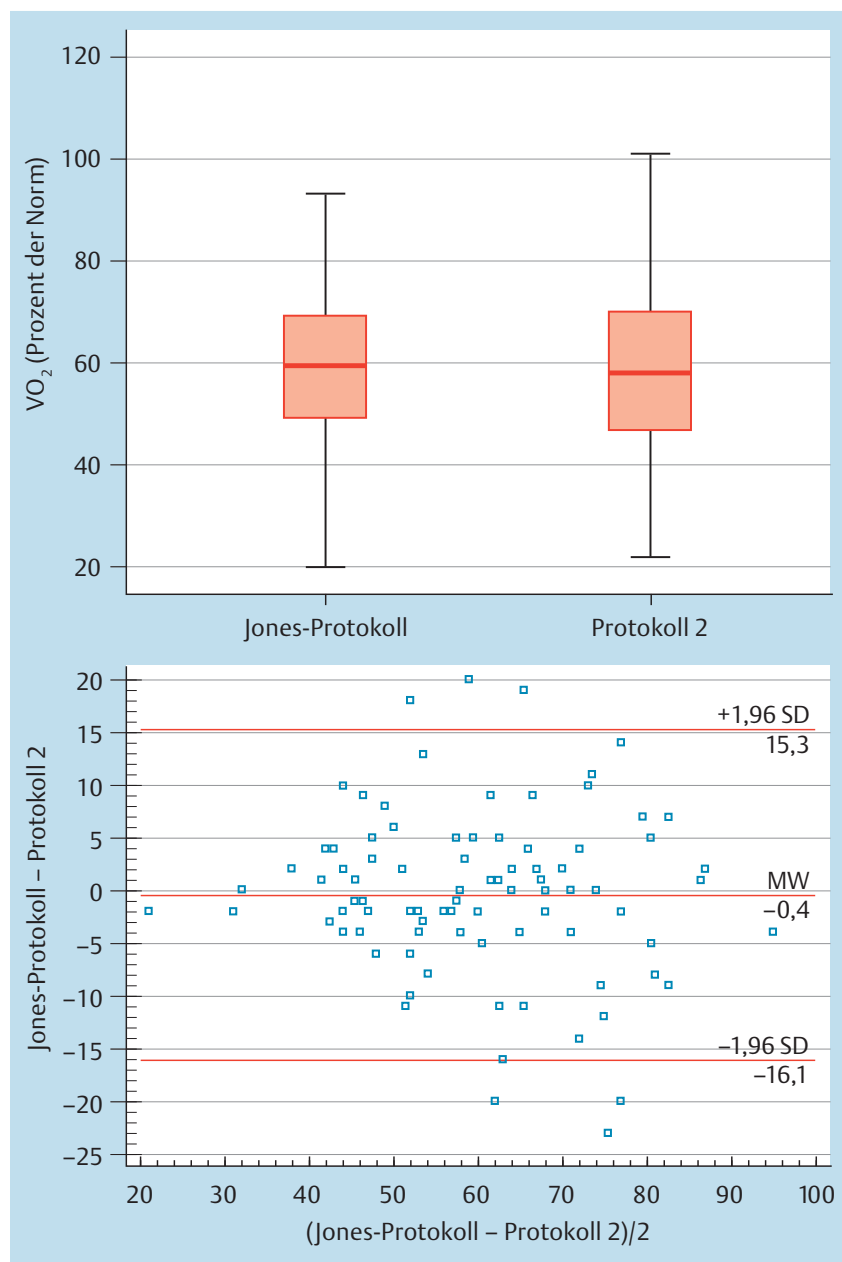

Abb.2 Darstellung der maximalen Sauerstoffaufnahme (in \% der Norm) mit beiden Protokollen als Boxplot bzw. Bland-Altman-Plot.

\section{Diskussion}

\section{$\nabla$}

Die vorliegenden Daten zeigen, dass nach Schulung des Personals und durchgeführter Zertifizierung in pneumologischen Facharztpraxen Patienten mit COPD einschließlich höherer Schweregrade sicher und reproduzierbar mittels CPET untersucht werden können. Dies wird durch die wiederholte Durchführung zweier (innerhalb von sieben Tagen) CPET belegt. Bei keiner der Untersuchungen kam es zu klinisch relevanten Komplikationen.

Die individualisierte Durchführung mit einer Untersuchungsdauer von 6-12 Minuten sowie die Anforderungen an die Genauigkeit der Messung der CPET-Daten sind weitgehend akzeptiert [26]. Für Gesunde $[27,28]$ sowie unterschiedliche Patienten z.B. mit chronischer Herzinsuffizienz [29], mit pulmonaler arterieller Hypertonie [30] oder COPD [14] konnte jedoch gezeigt werden, dass auch kürzere Untersuchungszeiten keine signifikant unterschiedlichen Ergebnisse bezüglich Ventilation, Herzfrequenz und Gasaustausch erbringen. So wurden bei COPD-Patienten mittels einer 16-Watt-Rampe (Steigerung jede Minute) Belastungszeiten um 4,0 (95\% CI 3-5 min) Minuten, bei einer 8-Watt-Rampe 6,6 (95\% CI 5-9 min) Minuten und bei einer 4-Watt-Rampe 8,7 (95\% CI 4-13 min) Minuten gemessen. Die CPET-Daten zeigten keine signifikanten Unterschiede, wobei die maximale Leistung mit zunehmenden Belastungsschritten anstieg [12]. Auch für einminütige Stufenprotokolle konnte gegenüber Rampenprotokollen gezeigt werden, dass unterschiedliche Belastungszeiten keinen Einfluss auf relevante spiroergometrische Parameter bei COPD-Patienten hatten [11,14]. Bei Rampentesten mit verschiedenen Steigerungsraten ( 5 vs. 10 vs. 20 Watt $/ \mathrm{min}$ ) konnte bei COPD-Patienten gezeigt werden, dass keine Unterschiede für die spiroergometrischen Parameter resultierten, obwohl sich die erreichte Leistung und die Belastungszeit signifikant unterschieden [15]. Auch unsere Daten zeigten diesen Effekt, wobei in Übereinstimmung mit der genannten Arbeit bei größeren Steigerungsstufen die Beziehung zwischen Leistung und Belastungszeit steiler wurde. Bei COPD-Patienten konnte zudem bei einem Vergleich zwischen einem Fahrrad-Rampenprotokoll (10 Watt Steigerung jede Minute) gegenüber einem Lauf- 


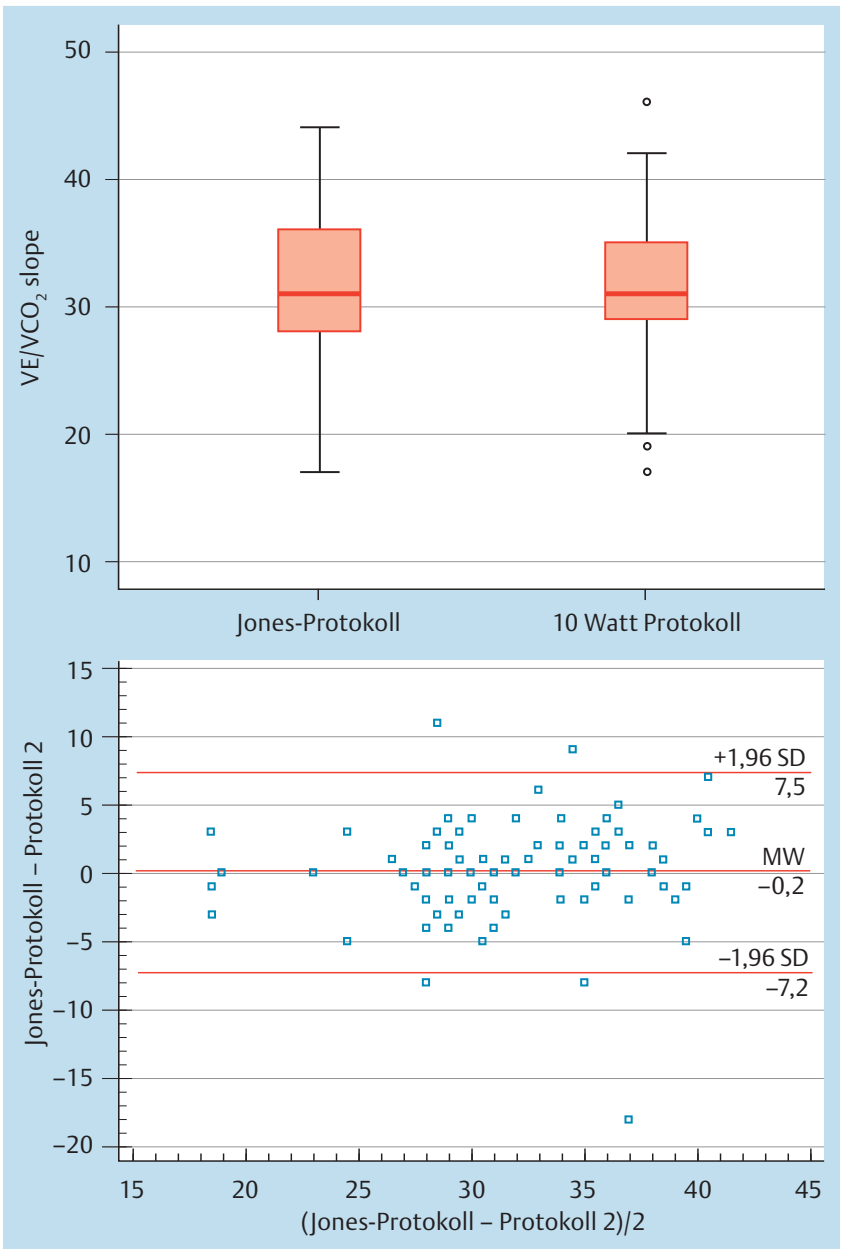

Abb. 3 Darstellung der Atemeffizienz (als $\mathrm{VE} / \mathrm{VCO}_{2}$ slope) mit beiden Protokollen als Boxplot bzw. Bland-Altman-Plot.

bandprotokoll (alle 2 Minuten Steigerung um 2,5\% Steigungswinkel) kein Unterschied bei der maximalen Ventilation, Herzfrequenz oder maximaler Sauerstoffaufnahme nachgewiesen werden [31]. Dagegen zeigten andere Untersuchungen mit COPD-Patienten eine geringe, aber jeweils signifikant höhere Sauerstoffaufnahme (bei gleichen Werten der Ventilation und des Gasaustausches) bei Anwendung des Laufbandes gegenüber dem Fahrrad [32-34].

Wir können anhand unserer Untersuchung sowohl eine Zunahme der maximalen erreichten Wattleistung bei größerem Belastungsanstieg (16 vs. 10 Watt/Minute) als auch eine geringe Belastungszeit bei größerem Belastungsanstieg zeigen. In der Gesamtgruppe wiesen die relevanten spiroergometrischen Befunde (Ventilation, Herzfrequenz, Blutdruck) zwischen beiden untersuchten Protokollen keine signifikanten Unterschiede auf. Lediglich bei einigen Parametern des Gasaustausches $\left(\mathrm{paO}_{2} ; \mathrm{AaDO}_{2}\right)$ zeigten sich signifikante Unterschiede, was auf eine unterschiedliche periphere Ausschöpfung hinweisen könnte.

Das Jones-Protokoll als Rampentest für die Fahrradbelastung gewährleistet valide und mit anderen Protokollen weitgehend vergleichbare Aussagen zu Leistung, Ventilation und Gasaustausch bei Patienten mit COPD. Da hierfür aktuelle, regional erhobene Normwerte [20] vorliegen, kann eine Anwendung zur Beurteilung der kardiopulmonalen Leistungsfähigkeit empfohlen werden.
Limitationen: Durch den konsekutiven Einschluss der Patienten in den fünf Praxen waren die Subgruppen (GOLD-Stadien III und IV) nicht gleichmäßig verteilt. Es wurde keine Randomisierung hinsichtlich des zuerst absolvierten Protokolls vorgenommen.

\section{Danksagung}

$\nabla$

Die Durchführung dieser Arbeit wurde ermöglicht durch die finanzielle Unterstützung der Firma AstraZeneca GmbH, Wedel, Deutschland, und des Kompetenz-Netzwerks Asthma/COPD, FKZ 01GI0881-0888, Ministerium für Bildung, Forschung und Kultur, Mecklenburg-Vorpommern, Deutschland.

\section{Interessenkonflikt \\ $\nabla$}

G. Hoheisel hat Erstattung von Reisekosten und Teilnahmegebühren für Kongresse, Honorare für Vorträge und finanzielle Zuwendungen für die Teilnahme an einer Studie erhalten.

S. Gläser hat Erstattung von Reisekosten und Teilnahmegebühren für Kongresse sowie Honorare für Vorträge erhalten.

H. Schrader hat Erstattung von Reisekosten und Teilnahmegebühren für Kongresse erhalten.

P-U. Haase hat Erstattung von Reisekosten und Teilnahmegebühren für Kongresse, Honorare für Vorträge, finanzielle Zuwendungen für die Teilnahme an einer Studie und für Beratertätigkeit erhalten.

R. Ewert hat Erstattung von Reisekosten und Teilnahmegebühren für Kongresse, Honorare für Vorträge und finanzielle Zuwendungen für die Teilnahme an einer Studie erhalten.

J. Winkler hat Erstattung von Reisekosten und Teilnahmegebühren für Kongresse, Honorare für Vorträge und finanzielle Zuwendungen für die Teilnahme an einer Studie erhalten.

A. Obst hat keine Interessenkonflikte angegeben.

\section{Institute}

Ernst-Moritz-Arndt-Universität Greifswald, Universitätsmedizin Greifswald, Klinik für Innere Medizin B (Direktor: Prof. Dr. med. S. B. Felix)

2 Pneumologische Praxis Leipzig

3 Pneumologische Praxis Burg

${ }^{4}$ Pneumologische Praxis Erfurt

${ }^{5}$ Pneumologische Praxis Halle

${ }^{6}$ Pneumologische Praxis Leipzig

\section{Literatur}

1 Palange P, Ward SA et al, ERS Task Force. Recommendations on the use of exercise testing in clinical practice. Eur Respir J 2007; 29: 185-209

2 Balady GJ, Arena R, Sietsema K et al. Clinician's Guide to cardiopulmonary exercise testing in adults: a scientific statement from the American Heart Association. Circulation 2010; 122: $191-225$

3 Cazzola M, MacNee W, Martinez FJ et al. Outcomes for COPD pharmacological trials: from lung function to biomarkers. Eur Respir J 2008; 31 : 416-469

4 O'Donnell DE, Flüge T, Gerken F et al. Effects of tiotropium on lung hyperinflation, dyspnoea and exercise tolerance in COPD. Eur Respir J 2004; 23: $832-840$

5 O'Donnell DE, Voduc N, Fitzpatrick $M$ et al. Effect of salmeterol on the ventilatory response to exercise in chronic obstructive pulmonary disease. Eur Respir J 2004; 24: 86-94

6 Ferrazza AM, Martolini D, Valli G et al. Cardiopulmonary Exercise Testing in the Functional and Prognostic Evaluation of Patients with Pulmonary Diseases. Respiration 2009; 77: 3-17 
7 Oga T, Nishimura K, Tsukino $M$ et al. Analysis of the factors related to mortality in chronic obstructive pulmonary disease: role of exercise capacity and health status. Am J Respir Crit Care Med 2003; 167: $544-549$

8 Hiraga T, Maekura R, Okuda Yet al. Prognostic predictors for survival in patients with COPD using cardiopulmonary exercise testing. Clin Physiol Funct Imaging 2003; 23: 324-331

9 Tojo $N$, Ichioka $M$, Chida $M$ et al. Pulmonary exercise testing predicts prognosis in patients with chronic obstructive pulmonary disease. Intern Med 2005; 44: 20-25

10 Preisser AM, Ochmann U. Die Spiroergometrie in der arbeitsmedizinischen Eignungsuntersuchung und Begutachtung. Pneumologie 2011; 65: $662-670$

11 Revill SM, Beck KE, Morgan MD. Comparison of the peak exercise response measured by the ramp and 1-min step cycle exercise protocols in patients with exertional dyspnea. Chest 2002; 121: 1099-1105

12 Benzo RP, Paramesh S, Patel SA et al. Optimal protocol selection for cardiopulmonary exercise testing in severe COPD. Chest 2007; 132 : $1500-1505$

13 Hsia D, Casaburi R, Pradhan A et al. Physiological responses to linear treadmill and cycle ergometer exercise in COPD. Eur Respir J 2009; 34: $605-615$

14 Debigaré $R$, Maltais F, Mallet $M$ et al. Influence of work rate incremental rate on the exercise responses in patients with COPD. Med Sci Sports Exerc 2000; 32: $1365-1368$

15 Miyahara $N$, Eda R, Takeyama $\mathrm{H}$ et al. Cardiorespiratory responses during cycle ergometer exercise with different ramp slope increments in patients with chronic obstructive pulmonary disease. Intern Med 2000; 39: 15-19

16 Vogelmeier C, Buhl R, Criée CP et al. Leitlinie der Deutschen Atemwegsliga und der Deutschen Gesellschaft für Pneumologie und Beatmungsmedizin zur Diagnostik und Therapie von Patienten mit chronisch obstruktiver Bronchitis und Lungenemphysem (COPD). Pneumologie 2007; 61: e1-e40

17 American Thoracic Society. Lung function testing: selection of reference values and interpretative strategies. Am Rev Respir Dis 1991; 144: $1202-1218$

18 American Thoracic Society. Standardization of Spirometry, 1994 Update. Am J Respir Crit Care Med 1995; 152: 1107-1136

19 Quanjer PH, Tammeling GJ, Cotes JE et al. Lung volumes and forced ventilatory flows. Report Working Party Standardization of Lung Function Tests, European Community for Steel and Coal. Official Statement of the European Respiratory Society. Eur Respir J 1993; 16: 5-40
20 Koch B, Schäper C, Ittermann $T$ et al. Reference values for cardiopulmonary exercise testing in healthy volunteers: the SHIP study. Eur Respir J 2009; 33: 389-397

21 Miller WF, Scacci R, Gast LR. Laboratory Evaluation of Pulmonary Function. Philadelphia: Lippincott Williams and Wilkins; 1987

22 Jones NL, Makrides L, Hitchcock $C$ et al. Normal standards for an incremental progressive cycle ergometer test. Am Rev Respir Dis 1985; 131: $700-708$

23 Wasserman K, Hansen JE, Sue DY et al. Principles of Exercise Testing and Interpretation: Including Pathophysiology and Clinical Applications. 4th: Ed. Philadelphia: Lippincott Williams and Wilkins; 2004

24 Brown SE, Fischer CE, Stansbury DW et al. Reproducibility of VO2max in patients with chronic air-flow obstruction. Am Rev Respir Dis 1985; 131: $435-438$

25 Cox NJ, Hendriks JC, Binkhorst RA et al. Reproducibility of incremental maximal cycle ergometer tests in patients with mild to moderate obstructive lung diseases. Lung 1989; 167: 129-133

26 Hansen JE, Sun XG, Yasunobu Y et al. Reproducibility of cardiopulmonary exercise measurements in patients with pulmonary arterial hypertension. Chest 2004; 126: 816-824

27 Midgley AW, Bentley DJ, Luttikholt $\mathrm{H}$ et al. Challenging a dogma of exercise physiology: does an incremental exercise test for valid VO 2 max determination really need to last between 8 and 12 minutes? Sports Med 2008; 38: $441-447$

28 Kang J, Chaloupka EC, Mastrangelo MA et al. Physiological comparisons among three maximal treadmill exercise protocols in trained and untrained individuals. Eur J Appl Physiol 2001; 84: 291 - 295

29 Myers J, Buchanan N, Walsh D et al. Comparison of the ramp versus standard exercise protocols. J Am Coll Cardiol 1991; 17: 1334-1342

30 Gläser S, Lodziewski S, Koch B et al. Influence of the incremental step size in work rate on exercise response and gas exchange in patients with pulmonary hypertension. BMC Pulm Med 2008; 8: 3

31 Mathur RS, Revill SM, Vara DD et al. Comparison of peak oxygen consumption during cycle and treadmill exercise in severe chronic obstructive pulmonary disease. Thorax 1995; 50: 829-833

32 Christensen CC, Ryg MS, Edvardsen A et al. Effect of exercise mode on oxygen uptake and blood gases in COPD patients. Respir Med 2004; 98: $656-660$

33 Buchfuhrer MJ, Hansen JE, Robinson TE et al. Optimizing the exercise protocol for cardiopulmonary assessment. J Appl Physiol 1983; 55 $1558-1564$

34 Hsia D, Casaburi R, Pradhan A et al. Physiological responses to linear treadmill and cycle ergometer exercise in COPD. Eur Respir J 2009; 34: $605-615$ 